\title{
Training Interpreters using Virtual Worlds
}

\author{
Panagiotis D. Ritsos ${ }^{1}$, Robert Gittins ${ }^{1}$, Sabine Braun ${ }^{2}$, \\ Catherine Slater ${ }^{2}$, and Jonathan C. Roberts ${ }^{1}$ \\ 1 Bangor University, Bangor, UK \\ \{p.ritsos, rgittins, j.c.roberts\}@bangor.ac.uk \\ 2 University of Surrey, Surrey, UK \\ \{c.slater, s.braun\}esurrey.ac.uk
}

\begin{abstract}
With the rise in population migration there has been an increased need for professional interpreters who can bridge language barriers and operate in a variety of fields such as business, legal, social and medical. Interpreters require specialized training to cope with the idiosyncrasies of each field and their potential clients need to be aware of professional parlance. We present 'Project IVY'. In IVY, users can make a selection from over 30 interpreter training scenarios situated in the $3 \mathrm{D}$ virtual world. Users then interpret the oral interaction of two avatar actors. In addition to creating different 3D scenarios, we have developed an asset management system for the oral files and permit users (mentors of the training interpreters) to easily upload and customize the 3D environment and observe which scenario is being used by a student. In this article we present the design and development of the IVY Virtual Environment and the asset management system. Finally we make discussion over our plans for further development.
\end{abstract}

Keywords: Interpreting, Virtual Environments, Second Life, Usability

\section{Introduction and Motivation}

Nowadays, interpreters are being called to master an ever broadening range of interpreting scenarios and skills, and are required to help in demanding situations such as courts, police interviews and medical emergencies. However, training for such situations can be challenging to do through traditional teaching methods. In addition, efforts to educate potential clients of the realities of working with an interpreter are scarce and normally separate from interpreter education.

In this paper we present the IVY Virtual Environment (IVY-VE). The aim of IVY is to address these needs, offering future interpreters (trainees) and future users of interpreters (clients) a 3D virtual environment that supports the acquisition and application of skills required in interpreter-mediated communication, focusing on the practice of consecutive and liaison interpreting in business and community settings. In contrast to many other initiatives in interpreter training, IVY attempts to integrate the education of interpreters and their clients closer together by employing working modes which allow the two user groups to engage with the environment in a variety of ways. 
The specific aims of project IVY are to develop (i) an adaptive 3D virtual environment for interpreting students and future users of interpreters; (ii) a range of virtual interpreting scenarios (e.g., 'business meeting') that can be run in different modes (e.g., 'interpreting mode', where interpreting students can practice using dialogues and monologues as shown in Fig. 1; 'exploration mode', where clients can learn about interpreting; 'live interaction', where both groups can engage in role plays);(iii) integrate into the virtual environment multilingual audiovisual content based on video corpora from the LLP project BACKBONE [1]; (iv) develop two sets of learning activities, one for students and one for clients (e.g., interpreting and awareness-raising exercises).

IVY-VE supports these aims by fusing web-based technologies with Second Life (SL). The concept is to abstract the 3D world (SL) from the assets (the audio and video files and management thereof). This is achieved through web technologies. This offers an intuitive means to manage scenarios and resources. We follow an Agile implementation strategy [2] where Interpreters and computer Programmers work closely together to develop several prototypes (each building on the previous), and evaluate the prototype at each iteration. In this article we also describe a critical evaluation of IVY-VE and discuss our findings.

This article is an extension of a conference publication [3]. In this publication we provide (1) more detailed description of the server-side mechanisms of IVY-VE, including audio playlists and file name conventions. (2) Add greater detail on the functionality of the in-world interface, especially to explain the different modes and operations. (3) Describe a usability evaluation of IVY. In fact, the evaluation focused on expert users, with experience in Virtual Learning Technology, developing Virtual Worlds, computer programming, and computer science research at postgraduate level.

The structure of the paper is as follows: Sect. 2 presents related work of training in Virtual Worlds, technology based interpreting training and the use of web technologies with virtual worlds, Sect. 3 describes several alternative design strategies that we considered, Sect. 4 explains in detail our implementation, Sect. 5 presents a usability evaluation from virtual world and computer-aided learning experts, Sect. 6 discusses our prototypes current limitations and outlines future work and Sect. 7 presents our conclusions.

\section{Related Work}

Different technologies have been used in training for many years. Technologies such as audio and video streaming, radio, web pages and wikis have all enabled remote learning to occur. Some of these can be used by several users synchronously, while others provide asynchronous learning environments where students can learn remotely and at their own speed. Many of these Information and Communication Technologies (ICT) enable blended learning experiences [4] that fuse face-to-face experiences with remote (web) technologies.

In addition, computer games and virtual environments have become a huge industry and educators have been investigating how these systems can be used for 
training. These so-called serious games [5] have a strong appeal to users [6,7] and seem to enhance participant involvement when used for educational and training purposes [8]. A comprehensive presentation of virtual worlds, their history and availability to date is beyond the scope of this paper; the reader can obtain such information from other sources such as Bartle [9] and Duncan et al. [10]. Instead, we focus on (i) ICT-based Interpreter Training, (ii) Pedagogy of Education and training in Virtual Worlds, and (iii) the fusion of web technologies with virtual worlds to provide a remote management.

\subsection{ICT-based Interpreter Training}

While virtual worlds have not been adopted in interpreting training programs, other Information and Communication Technologies (ICT) have been successfully used. These systems allow the trainee interpreters to practice and also develop computing literacy skills, which is becoming increasingly important in the interpreter's daily work [11].

Many of the training tools use audio files. The SIMON (Shared Interpreting Materials Online) project [12] contains a database of mp3 audio recordings and transcripts as pdf files. The SIMON project classified the material according to progression level, exercise type language and thematic domain, which helps the users improve their skills and enables materials to be shared between educators.

Video is also used in interpreter training. Hansen and Shlesinger [13] describe a video-based interpreter training initiative. The video clips show tutors and other actors performing monologues and bilingual role-play dialogues. Similarly, the English Language Interview Corpus as a Second-Language Application (ELISA) [14] is a collection of video interviews with native speakers of English which is designed to be a resource for language learning and interpreter training. ELISA was a forerunner of BACKBONE [1] which produced video interviews with native speakers of English, French, German, Polish, Spanish and Turkish, as well as with non native speakers of English. The BACKBONE resource also includes interpreting exercises that can be applied to any of the videos in the BACKBONE corpora.

Apart from serving audio and video materials to the user, another focus is on authoring. Sandrelli and Jerez [15] describe the evolution of authoring programs for interpreters, starting with Interpr-IT, through Interpretations, and finally to Black Box 3.0. They highlight functionalities such as a voice recording feature, on-screen note-pad, pitch-tracker for gauging performance etc. While their focus was on authoring, the need to manage resources is an important challenge in the development of these training systems, and is thus a major feature of our IVY environment.

\subsection{Pedagogy of Education and Training in Virtual Worlds}

Today's virtual worlds and massively multiplayer online games (MMOGs) are descendants of Multi-user virtual environments (MUVEs) [9,16] of the 1980s. 
Second Life is a popular commercial virtual world, often employed by educational institutions [17]. It is used in various fields such as medical and health education [18], environmental education [19] and security [20]. Another virtual world that has attracted the attention of researchers $[21,22,23]$ is Activeworlds launched in 1997 which includes a dedicated educational world, called Educational Universe (AWEDU). OpenSimmulator is a newer virtual world implementation, in the form of an open source multi-platform, multi-user 3D application server. It is almost exclusively used by universities and big companies and has recently attracted the attention of researchers [24,25].

The pedagogic implications of using Virtual Worlds in training and simulation scenarios have been investigated by many researchers. Savin-Baden et al. [26] argue that "technology has led pedagogy" and further technological development should be driven by pedagogy itself. Thackray et al. [27] suggest that different methodologies are used among institutions, disciplines and instructors, which introduces much variety on virtual world implementations and associated practices.

More importantly, Salmon et al. [28] point to challenges of 'familiarity'. Users unfamiliarity with the world increases challenges inherent to the learning process [29]. In addition student expectations can also effect the learning process. Moschini [30] highlights that users of such environments expect interactive content, rather than a mere passive learning experience. Consequently it is important to structure the tasks appropriately and make them intuitive, especially considering that Second Life can be chaotic and anarchic, as claimed by Carr et al. [29].

Evaluation of the learning environment is obviously an important aspect in any system development. For instance, Tymczynśka [31] describes how the Moodle Learning Management system[32] was integrated into a healthcare interpreting module at Adam Mickiewicz University in Poland. She highlights five different criteria for evaluating the applicability of the learning resources - student learning potential, student and context fit, authenticity, practicality and sense of achievement. These are similar to the findings from the pedagogical considerations emerging from other virtual world projects. However, while researchers are considering the potential positive impact on the broader serious games learning environments [33], none of the studies reported have researched whether the ability to learn improves in virtual worlds. An equally important aspect is that studies to date have predominately focused on students in a higher education environment.

\subsection{Web technologies and Virtual worlds}

One of the drawbacks of most virtual environments is that they are exclusive [34] because they don't have the functions necessary to interact or operate with other learning environments. In addition they often require 'in-world' scripting, even for the simplest examples of communication and mediation. Therefore managing content in these worlds requires knowledge of proprietary scripting languages such as LSL (Linden Scripting Language) used in Second Life. However these 
languages do not have the features of established web technologies like Java, or JavaScript etc. and can thus limit the functionality of potential implementations; especially those requiring frequent content management and material updates.

Some researchers have investigated how virtual worlds and web technologies merge [35] to manage the content better and enable a better in-world experience. For example, Sloodle [36] fuses Second Life with Moodle [32]. It presents information in Second Life using HTTP-requests that communicate with Moodle's PHP-based database front end. Zender et al. [34] present their SOA-based approach, using Web Services to provide lecture streaming and a messaging service in Second Life.

But there are important limitations in establishing communication between Second Life and external entities (e.g., scripts, programs). LSL uses functions that utilize HTTP and XML, such as IlHTTPRequest or the older RPC-based lisendRemoteData and llRemoteDataReply for this purpose. However, Linden imposes time delays and limits on these methods, such as request size, number of requests per object, per owner and per period of time, making more demanding communication tasks quite difficult to implement.

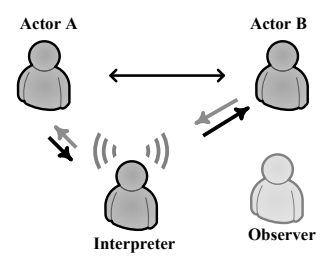

(a) Dialogue scenario

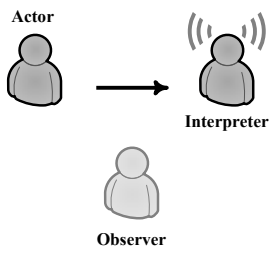

(b) Monologue scenario

Fig. 1. A range of scenarios is created in IVY VE to support interpreting practice in ready-made dialogues (a) and monologues (b) as well as live interaction and other working modes. In the dialogues and monologues, the actors are avatars controlled by the environment (robots), representing the interlocutor(s) in each scenario, and the avatar of the interpreting student. In live interactions, avatars of interpreting students and their 'clients' interact.

\section{IVY Design}

Our design vision was to create a virtual world where interpreters can be immersed in different scenarios, listen to virtual actors and interpret the conversations. Users need to choose a scenario from a menu of choices, navigate to a room or location that has appropriate appearance and follow exercises and view instructions. Mentors also need to be able to populate the world with training materials, upload audio tracks for the actors and manage the world assets. 
Therefore, our first strategic choice concerned the virtual world. We chose to use Second Life (SL), instead of other virtual worlds (such as OpenSimulator), for several reasons. SL provides a large community of users, developers and enthusiasts. These users have created many add-ons, plugins and therefore there are many examples of customizations and vast support for scripting and assets for creating the scenario settings. This is extremely useful to bootstrap the development of a virtual learning environment. SL is a platform for social interaction and education, used by numerous institutions, colleges, universities - thus through using SL it will increase the chances of exposure and information dissemination of the IVY project. Finally, SL is a managed service with public servers; subsequently, prototypes could be quickly built and shared between the developers and partners in IVY.

With the decision to use Second Life two alternative implementation strategies were considered. The main decision was whether the IVY-VE interface would be scripted in Second Life, using LSL or implemented as a separate, external component using popular web technologies such as HTML/CSS and Java.

The major advantage of the first solution is that the code can closely integrate with other objects in the scenes, and menus and GUI elements built using LSL have comparable aesthetics to the main client GUI. Embedding scripts into objects is common practice and many information display systems make use of such mechanisms. Nonetheless, due to the static nature of including data into scripts it would be time-consuming to update the menu. LSL supports database connections through PHP scripts, as used by some researchers, but the solution is limited in terms of the quantity of information that can be sent or received.

We chose to follow the second approach. This has the advantage of relying on popular and established techniques for building web applications. The level of menu customization and enrichment, database connectivity and overall flexibility surpasses that of LSL-based scripting. However, this implementation requires either embedding an SL viewer on a web page or a web-like, in-world interface. Unfortunately, despite the popularity of the notion of web-based SL viewers, Linden Labs does not officially offer a web client and most of the efforts to implement something similar seem to have ceased. Canvas, from Tipodean Technologies ${ }^{1}$ appears to be the only implementation of such a viewer, claiming to support both SL and OpenSim.

\section{Implementation}

Our chosen implementation strategy therefore is a hybrid solution (see Fig. 2). The scenario manager is independent of SL and is used by content-managers to manage scenarios and user's information, offering basic CRUD functionality. The second part is viewable within Second Life, in the form of a heads-updisplay menu (HUD). The information displayed on the HUD is populated from a database and depicts available scenarios to the user. It includes the audio player and methods to initiate in-world teleport events.

\footnotetext{
${ }^{1}$ http: / / www. tipodean. com/
} 


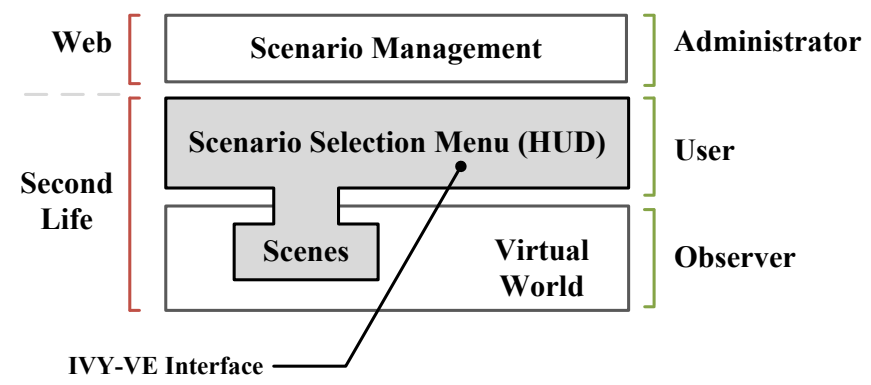

Fig. 2. IVY-VE Architecture - depicting the three main parts of the application: (i) the web-based administration panel, (ii) the in-world HUD, and the scenes associated with the scenarios and the remaining, (iii) the virtual world (SL).

Nonetheless, a major consequence of using SL is the lack of mechanisms such as instancing, replication and zoning, used in massively multiplayer online games (MMOGs)[37] to allow an increasing number of simultaneous users to be present in the game/virtual world. Contemporary MMOGs, such as EVE ${ }^{2}$ and World of Warcraft use multiserver architectures to allow access to many users at a given time. However SL is fairly static when it comes to each individual island and the virtual world created on it, resulting in an environment with almost no dynamic scalability.

Of particular interest in our research is instancing; the creation of multiple copies of parts and locations of the virtual world. Several of the interpreting scenarios take place in the same setting, e.g., many language combinations take place in a classroom. Instancing these rooms would allow the world to scale according to users' demands. While it is possible to copy settings, this method has resource implications. Indeed due to limits on the quantity of primitives available to the IVY Island we cannot merely replicate all models in different points on the SL island.

To combat these limitations we use a collection of unique, in-world locations for each type of scenario (e.g., Classroom, Office). We replicate some commonly used models, but importantly lockout the scenarios on the menu when all possible locations are in use. Once the user exits the selected scenario, the setting becomes available. While Holodeck or rez-on-demand scripts exist in SL we decided to maintain consistency in the virtual environment and explore such scalable solutions in future implementations. Fig. 3 shows the relationships and sequence of actions in IVY-VE.

\subsection{IVY Scenario Management}

The IVY scenario manager application was built using Appfuse $2^{3}$. Appfuse, built upon the Java platform, uses industry-standard features, such as Apache

\footnotetext{
2 http://www.eve-online.com/

${ }^{3}$ http: / / www. appfuse.org
} 


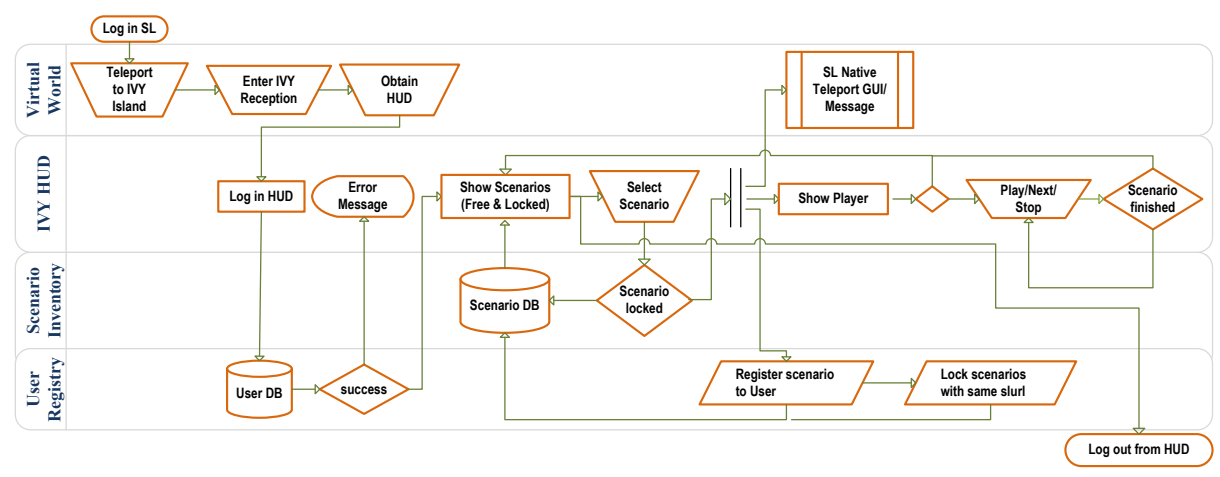

Fig. 3. IVY-VE Cross-functional flowchart - depicting the functions and the process flow, in each tier of the application, from log-in to dialogue selection and playback.

Maven integration, JPA support for database operations and popular web frameworks such as Spring MVC and Apache Struts, employed in this example. Our prototype is deployed using Apache Tomcat 6.x and uses the MySql 5.x database. Appfuse comes with the following features, used in IVY-VE:

- Generic CRUD backend, allowing the design and implementation of mechanisms for creating, uploading, editing and deleting scenarios and users.

- Authentication and authorization, allowing easy implementation of access control based on Spring Security.

- User management, enabling administrators (interpreting tutors) to easily manage the pool of users (students) and their access level.

- Strong Internationalization support, providing means of translating the web application in all the languages supported in our corpus.

\subsection{Scenario Locations and Actors}

The IVY environment was built on a full SL Island using several geometric models and textures. Photorealistic landscaping of both scenario locations and distant views provide a natural-looking environment for users to explore, and a series of buildings and rooms including a reception area, meeting rooms, classroom and offices have been built (see Fig. 4). The goal throughout the build was to use the smallest number of primitives to allow scalability and replication in the future.

The reception area serves as a central focus for visitors (Fig. 4a) where users can obtain the HUD, view introductory videos and read noticeboards with information. Care and effort has been put into the scale of objects [38].

In fact, there are two types of rooms that are used for the scenarios, that match the modes (as discussed in Sect. 1): those, used for the interpreting mode and those used in live mode.

The interpreting scenarios are actually located on sky platforms. These are modular components that can be readily replicated to expand capacity of a 


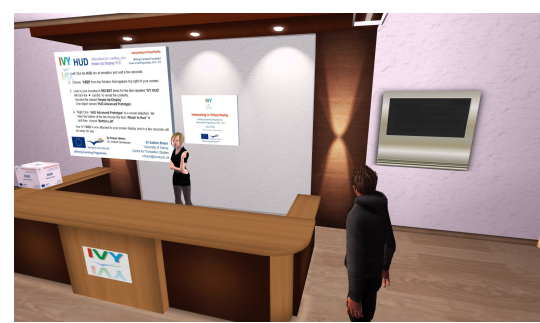

(a) IVY Reception

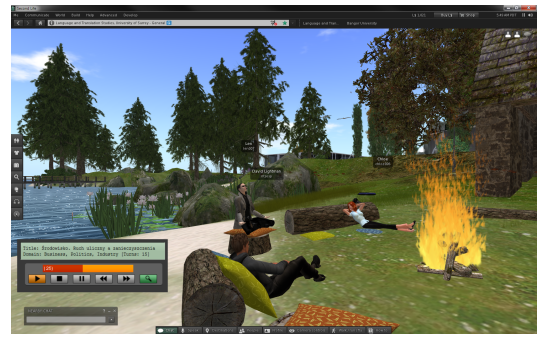

(c) Outdoor setting

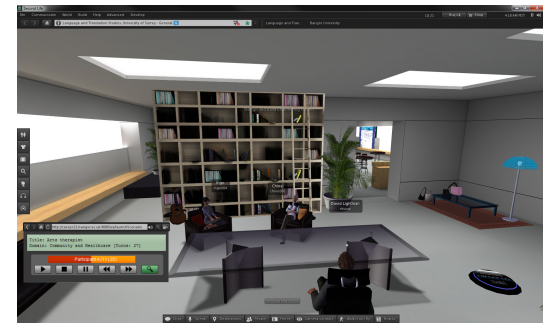

(b) Arts Therapy Room

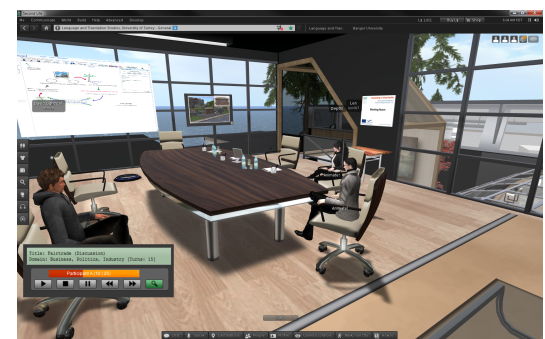

(d) Meeting Room

Fig. 4. Screenshots of the IVY-VE in use, showing scenario locations in Second Life The IVY Reception (a) is where visitors are initially teleported and where they pick the HUD from. The locations were designed to match the available scenario themes, maintaining a generic look as much as possible to allow re-use of primitives

particular scenario, depending on their frequency of usage in the corpus. Also by being located in the sky, users need to teleport to them through our menu, and it is less likely that they are accessed by merely walking to them.

Live mode locations, along with an exhibition hall used in the exploration mode are located on the Island floor. These are necessarily more accessible such that avatars can walk to them easily and thus permit more opportunities for social interaction.

Each scenario is populated with 'actors'. Currently we manually situate each of these SL 'robots' (bots) in specific locations, and match the male/female versions to the scenarios, adding another level of complexity to the management system. The ultimate aim is for the bots to teleport in-situ upon scenario selection, relay sound when it is their turn to speak and perform gesture animations related to the scenario. We use both Pikkubots ${ }^{4}$ and Thoys ${ }^{5}$ and control them from a separate server and use recursive animation overrides (AO) to give them movement.

The exhibition mode has a dedicated exhibition area on the IVY island. This area includes information and presentation boards. Different presentations are

\footnotetext{
4 http://www $\cdot$ pikkubot.de/

${ }^{5}$ http://slbot.thoys.nl/
} 


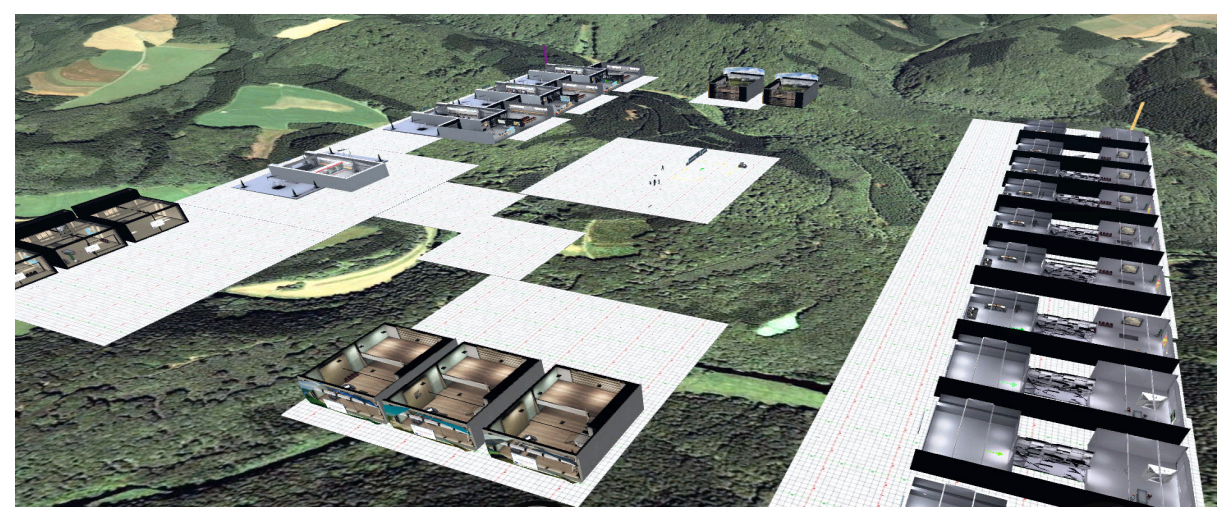

Fig. 5. Aerial view of the skyboxes, where the locations used for interpreting mode are placed. This view normally is not accessible to users, who are teleported directly into each location/room.

available including those using Microsoft Powerpoint presentations and others that use the HTML-on-a-prim feature, that maps HTML onto virtual objects. The latter are implemented to display textual information arranged in meaningful sections using a jQuery accordion menu, minimizing the need to excessive vertical scrolling. Various informative videos are also embedded on prims, using the same strategy, displaying webpages with embedded media.

\subsection{Audio File Management}

The IVY-VE uses audio extracts (segments), in MPEG-2 Audio Layer III format, from the LLP project BACKBONE, wrapped in XSPF (XML Shareable Playlist Format)[39] play-lists (scripts) and played within pre-fabricated scenes. Audio segments are uniquely named (see Fig. 6) and can be interchanged - within each script - to derive further language combinations of that scenario.

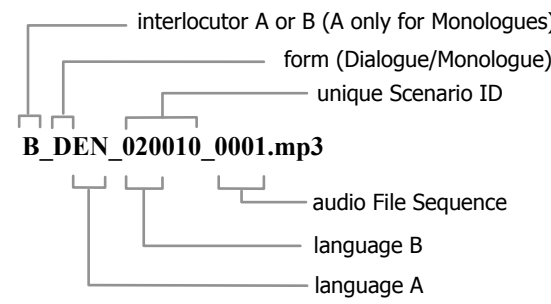

(a) Audio file naming

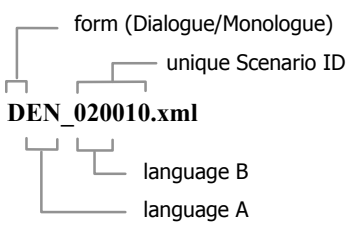

(b) Scenario Playlist naming

Fig. 6. Audio file and playlist naming convention. The name of each file is unique. Interchanging audio files yields additional language combinations of a particular scenario, which in turn are uniquely named in XSPF playlists. 


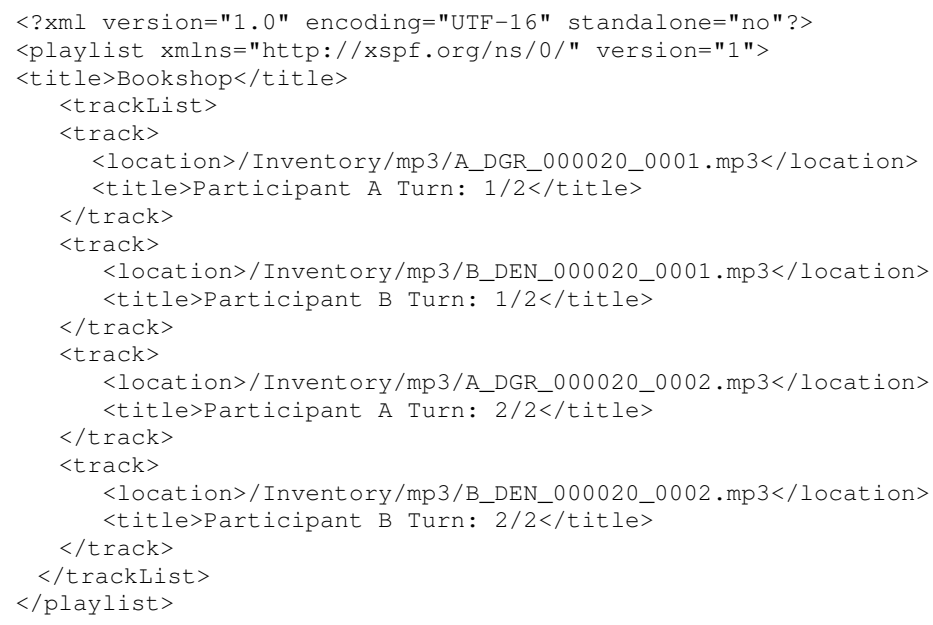

Fig. 7. Example of an IVY XSPF playlist, for a scenario about a Bookshop, where Interlocutor A speaks in Greek and Interlocutor B in English.

Each actor may speak for more than one consecutive segments, allowing for scenarios where one talks for extended periods. However, it is assumed that only one actor talks per audio segment and there is no overlap between actors' speech. Each scenario has textual information associated with it, such as brief content information, scene description and domain keywords. XSPF playlist are enhanced upon upload (see Sect. 4.5) with turn counters per interlocutor. The information is places in the XSPF element allocated for the title of each track, corresponding to each scenario turn (see Fig. 7).

\section{$4.4 \quad$ In-World Head-Up Display}

The HUD makes use of the HTML-on-a-prim feature of SL. The HUD (Fig. 8) is built using the jQuery JavaScript library, and displays the list of the scenarios from a database as a drill-down menu and placed at the lower left of the user's viewport. By relying on web technologies the text is displayed in-world in the best possible rendering quality, particularly when compared to text rendered on 'prims' using native LSL. Moreover, the extent of character sets supported using web technologies ensures that our system displays properly textual information in all character sets of languages in our corpus (which is particularly for different language combinations). The functionality embedded in the HUD includes:

Navigation Navigation through the island is achieved using slurls, providing direct url-like teleport links to locations within the virtual world. Each slurl is being called upon a series of events, such as scenario launch in interpreting mode, location selection in live-interpreting mode and launch of 


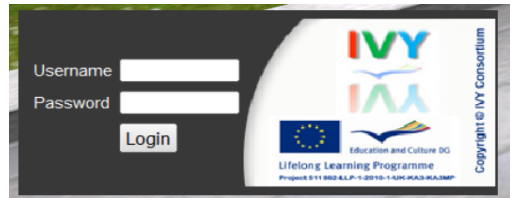

(a) Login Screen

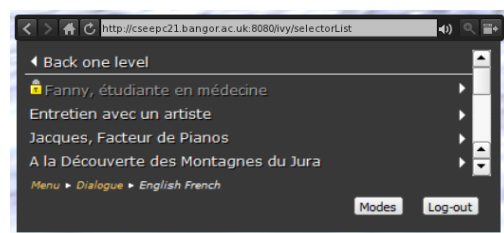

(c) Scenario Selection

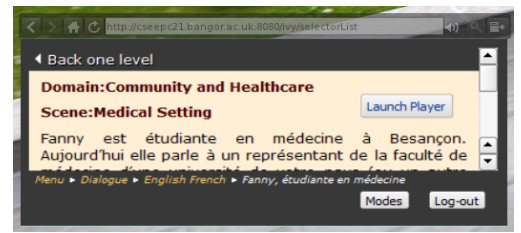

(e) Scenario Info

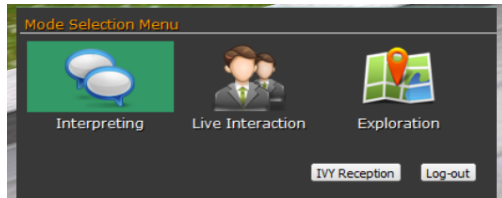

(b) Mode Selection View

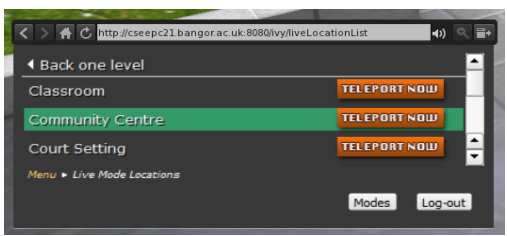

(d) Live Location Menu

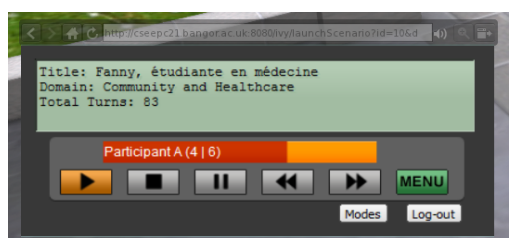

(f) Scenario Player

Fig. 8. Screen captures of the IVY-VE HUD, depicting the login screen (a), the mode selection view(b), the scenario title selection view (c), the live location selection view (d), the scenario information view (e) and the scenario player (f).

the exhibition mode, triggering the native SL-client teleport interface. Fig. 4 shows some screenshots of the IVY-VE.

Mode Selection Modes are selected in the Mode Selection View by means of icons.

Utility Buttons Log-off and Modes access buttons are available in all views, whereas a teleport launch button to IVY Reception is available in the Mode Selection View

Audio File Playback Audio is being played by means of a Flash player, parsing the XSPF playlist upon each dialogue selection. The player control resemble a standard audio player interface allowing users to play, pause, repeat and advance tracks, corresponding to each turn of the scenario.

Textual Information Each mode has textual information and icons to assist users. Particularly in the case of the interpreting mode, launching a scenario allows access to scenario information, in the form of a summary brief, keywords and location description.

Access to SL GUI functions (limited) A limited number of slurls, using the prefix secondlife:///app/, allows direct control of the native SL 


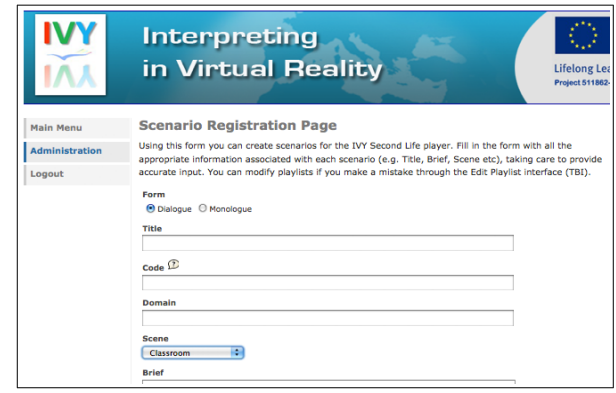

(a) Scenario Upload Form

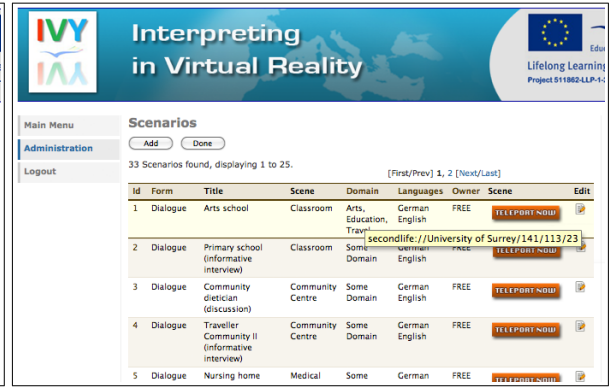

(b) Scenario Listing View

Fig. 9. The Administrator's panel includes tools to create, edit, delete scenarios and users as well as monitor scenario usage, launch SL client and teleport to scenario locations, using slurls.

client user interface ${ }^{6}$. We make use of such a slurl to launch the IVY Group inspection panel, displaying group members, allowing communication by chat, instant messaging as well as means of offering teleports on locations in the IVY Island.

\subsection{Administrator's Panel}

One of the most important functionality features of the IVY-VE is the scenario and user management console. Content administrators can create, edit and delete scenarios, through a form (shown in Fig. 9) where they provide textual information (title, language combinations, participants gender, scenario brief etc.), select the scenario location from a series of pre-fabricated scenes and provide an ordered file listing of the audio tracks of the scenario. Textual information is stored in the database and the audio file listing in the aforementioned XSPF playlist format, in the playlist inventory.

In addition, a listing of all the scenarios in the system's inventory allows tutors to see which user is going through which scenario at a given time and teleport to that location, by launching their local SL client. Administrators can choose to use the slurl links to launch their SL client and teleport to those locations. Particular attention was paid to extended language support of the forms and database, so that scenario information in different character sets can be entered and displayed properly.

\section{Usability Evaluation}

The IVY-VE prototype underwent a usability evaluation from experts in virtual environments and computer-aided learning technologies. The purpose of

\footnotetext{
${ }^{6}$ http://wiki.secondlife.com/wiki/Viewer_URI_Name_Space
} 
our evaluation was to assess the usability of IVY-VE, focusing on the virtual world and HUD. We chose to follow a formative evaluation approach [40]. The evaluation took place during the Virtual Learning Technologies 2012 workshop, in Bangor University.

\subsection{Evaluation Methodology \& Tasks}

The evaluation methodology was as follows. The participants were formally welcomed and presented with a project introduction, description of the functionality of the HUD and evaluation task overview. The roles of the test facilitators and evaluators was clarified, emphasizing that we were not evaluating the participants' performance but the usability of our prototype. Written consent for their participation was obtained from all participants. A video tutorial of the IVY-VE functionality was shown to the participants, describing each evaluation task and highlighting important features such as the teleporting.

Once the briefing was over, the participants used avatars and HUD logins provided by the test facilitators and were asked to go through the tasks. They were encouraged to work without guidance, unless they did not understand a task or the HUD's functionality, or were unclear of how to progress. Upon completion of the tasks the participants were asked to complete a questionnaire.

The questionnaire contained three parts. First to collect demographic information, then they answered questions from the System Usability Scale (SUS) [41], and finally they answered open-ended questions (shown in table Table 1) and gave general comments.

As Lindgaard and Chattratichart [42] demonstrated the number of issues discovered in a usability evaluation correlate significantly with the evaluation task coverage and not with the number of participants. It was important therefore to ensure that the tasks cover the complete functionality spectrum of the evaluated system, compared to having a large pool of participants. In that respect, four evaluation tasks were designed around obtaining the HUD from the IVY reception hub and going through all the functionality modes, i.e., interpreting, live interaction and exploration. All tasks were to be performed in Second Life, using the HUD, much like the end users would.

\subsection{Evaluation Conditions, Apparatus \& Participants}

The evaluation was carried out, for reasons of convenience and availability, using laptops, with Intel i5 CPUs, 8GB RAM and Nvidia NVS4200M GPUs. All laptops used the official SL client (v. 3.4.1 (266581)). Laptops were connected over a wireless connection to Bangor University's infrastructure. SL Client Lag meter indicated 'normal lag' throughout the experiment. We set the SL client's graphics settings to the lowest possible settings, as our evaluation did not focus on the quality of the locations and the resulting sense of immersion.

We used a convenience sample [43] of twelve participants, nine male and three female, between 23 and 59 years of age. Seven of them reported they have had little familiarity with interpreters, mostly from the vocational meetings, 
Table 1. Open-ended questionnaire, used in our IVY-VE evaluation

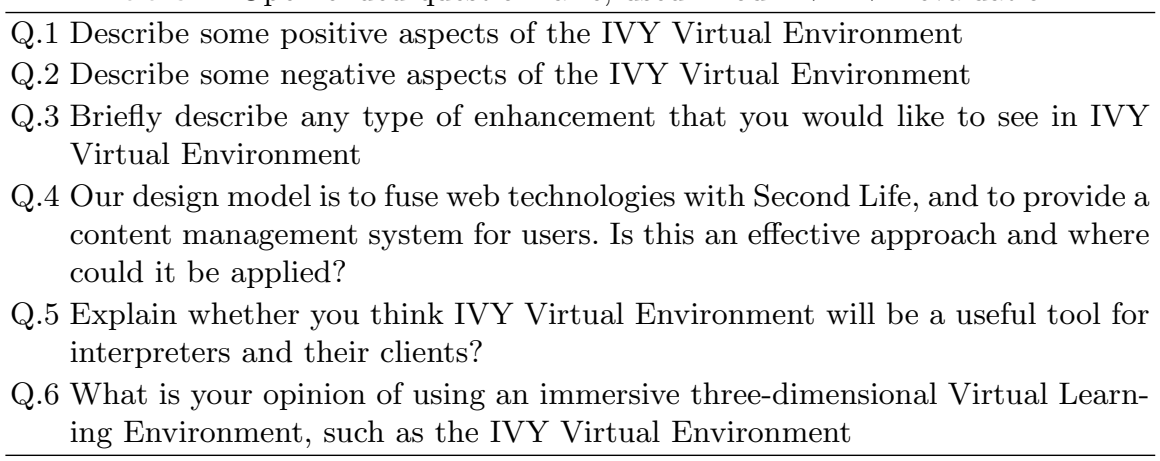

conferences and presentations. The remaining five reported no familiarity with interpreters and their practice. In addition we recorded their perceived expertise in computers, virtual worlds and environments, Second Life and computer games, shown in Fig. 10a.

\subsection{Results}

The average SUS score, rating overall usability, was $65.5 / 100$, with individual scores from participants ranging between 32.5 and 87.5. The scores for each participant are shown in Fig. 10a and we discuss answers to each question.

Q1,2. When participants were asked to list positive features of IVY-VE they commented favourably on the HUD interface and the "simplicity of controlling audio", the "multiple of different locations", the ease of selecting modes and scenarios and the good aesthetics of some locations. When participants were asked to list negative aspects five reported that "the lack of visual cues on who is speaking is an important limitation". In addition, two felt that IVY-VE is "not as interactive as modern games" and "needs a bit of training to get used to, mostly due to movement and interaction with objects (panels etc.) in SL .

Q3. Participants were also asked to discuss possible enhancements they would like to see in IVY-VE in the future. The most common enhancements included: better visual cues to assist interpreters on who is speaking, "a facility to record one's interpreting for future assessment" and that directional sound may enhance usability. One user mentioned that "the environment is not used in a dynamic way" (see Sect. 4) and that implementing such mechanisms would enhance usability. In addition, the same user argued that "getting an emotional connection with the situation, characters and narrative could make things more interesting, making situations tense or pressured etc.".

Q4. All participants expressed positively that the web technologies rendered 'text' better and could display different character sets (in-world text renderings are traditionally of low quality). More importantly, they expressed that a webbased scenario management system is the best way to update content in such a 


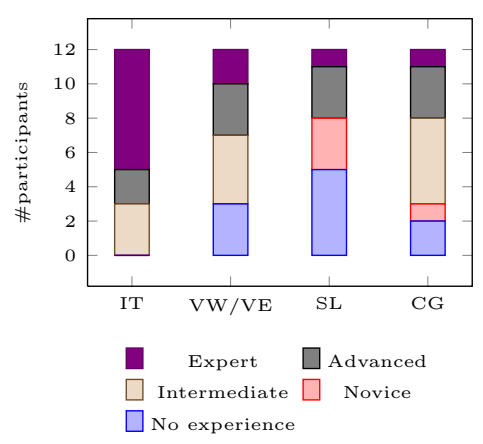

(a) Participant Profile

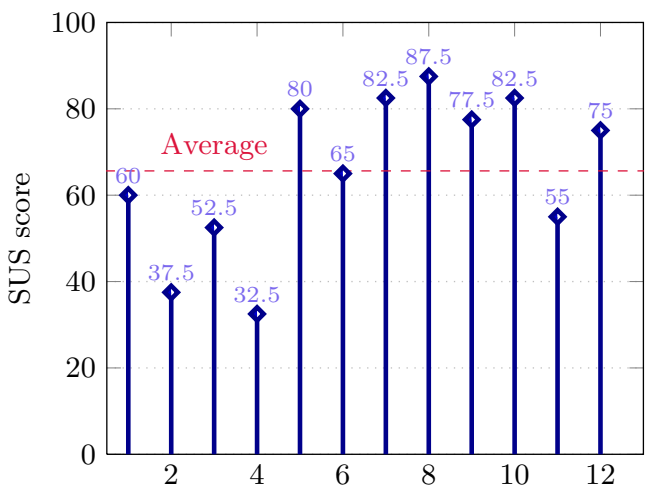

(b) SUS Scores

Fig. 10. Participant expertise in Computers in general (IT), Virtual Worlds and Environments (VW/VEs), Second Life (SL) and Computer Games (CG) is shown in Fig. 10a. System Usability Scores for each participant along with the average score is shown in Fig. 10b

system. However, controlling virtual assets and modifying the world aesthetics requires access to the world and can not be easily done using an external, say web-based, system.

Q5.Regarding the usefulness of such an environment for interpreters, most users felt that if the enhancements they suggested were implemented they system would be an effective tool of simulation and training. Some appeared sceptical on whether such systems would be popular with students as "they take some getting used too".

Q6. Finally participants saw much potential on the use of Virtual Learning Environments (such as IVY-VE) for training, particularly for situations which are hard to recreate with other means. Two of the participants argued that because computer games can be educating so can VLEs in general. Three participants pinpointed that as VLEs become more accessible on the web or through mobile devices they will be more common place and become traditional educational tools.

\section{Discussion}

The results of the usability evaluation as well as the preliminary functional assessment [3] were positive. The average score in our evaluation was $65.5 / 100$. This corresponds well with SUS scores of other web-based applications that have a mean score of 60 [44]. From this feedback we classify useful features and limitations of our current prototype, in five categories: scenario management, interface design, virtual world scalability, actor management and scenario execution. 
As far as scenario management is concerned our web-based scenario management system, compared to the alternative of scripting content in the world, arguably, ensures ease of access and good level of control from interpreting tutors with no programming experience. Additionally, relying on web technologies to render text properly and support all the character sets required in our corpus yields good results and is simpler than enabling such a feature in bespoke systems.

We developed the HUD interface using web technologies, such as HTML/CSS and jQuery. By separating the virtual world from the functionality of the HUD results in a very flexible solution. Scripting similar functionality using LSL would be possible but hard. In addition, our approach allows us to experiment in the future with various other designs and explore the resulting user experience. There are certainly improvements that can be made, such as improving the visual cues to highlight the current speaking actor. But, as most evaluators mentioned, the current HUD was easy to use, informative and provided good control for teleporting and choosing modes and scenarios

Scalability issues are still a concern (see the discussion in Sect. 4) especially over zoning, instancing and replication. Our solution works exceptionally well, but we have a limit on the number of such scenarios the SL island can support. Moreover, actor management is affected, both in terms of populating scenarios as well as making them more interactive.

Finally, the execution and use of SL also provides challenges of quality. Newer modern computer games include high quality renderings with complex shadows, physics engines and caustic light models. Overall, it is evident that SL introduces important limitations, despite being relatively straightforward to develop and create an operational prototype.

\section{Conclusions}

In this article we present the design, implementation and evaluation of IVY-VE, a 3D virtual environment that supports the acquisition and application of skills required in interpreter-mediated communication.

One of our overarching successes is the separation of the content and manipulation of the learning material from the virtual world. This paradigm has many benefits: not only can the educator easily upload materials to a web interface, but the abstraction affords extensibility (where a new virtual reality environment could be used instead). The system is based on the fusion of Second Life and web technologies such as HTML/CSS, Appfuse, jQuery and JavaScript has been successful.

We have performed a usability evaluation (based on the SUS methodology), and have collected feedback from experts and researchers of Virtual Worlds and Computer Science. The evaluation of the work has been positive. Indeed, by following the SUS methodology we can compare the usability of this system to the scores of similar systems [44]. With a SUS score of 65.5/100 our usability compares favourably to other web-based tools. 
We discuss various limitations with IVY-VE in this article. In fact, many of these limitations could be overcome by developing a bespoke virtual environment such as by using a game engine (such as Unity), and other features such as directional and localized sound, more elaborate actor movement and interaction and in-world communication and collaboration could be included in a single application. We could use our current scenario management system. Indeed other modalities such as haptics [45]) could be added to the environment to develop a more realistic interactive experience. However, the use of SL has several advantages: it enables other views to readily access the world, enables quick building of rooms and buildings

In conclusion, we have developed a usable virtual environment for interpreting students and clients and our evaluation of the tool suggests it to be useful and valuable. However more work is required to develop IVY further, as a bespoke system, with enhanced scalability and tighter integration to the virtual world, allowing us to investigate further the resulting sense of presence. Our aspiration is to explore such a solution, based on the findings of using the current prototype and the feedback that we have collected, regarding the associated user experience of interpreter students and clients.

\section{Acknowledgements}

We are grateful to all the members of the IVY consortium, for their assistance and contribution to the project. This work was supported by the European Commission through project 511862-LLP-1-2010-1-UK-KA3-KA3MP in the Lifelong Learning Programme.

\section{References}

1. Kohn, K., Hoffstaedter, P., Widmann, J.: BACKBONE - Pedagogic Corpora for Content \& Language Integrated Learning. In: Eurocall Conference Proceedings, Macmillan ELT (2009)

2. Highsmith, J.: Agile software development ecosystems. Addison-Wesley Longman Publishing Co., Inc. (2002)

3. Ritsos, P.D., Gittins, R., Roberts, J.C., Braun, S., Slater, C.: Using virtual reality for interpreter-mediated communication and training. In: Cyberworlds (CW), 2012 International Conference on, IEEE (2012) 191-198

4. Bonk, C., Graham, C.: The handbook of blended learning: Global perspectives, local designs. Pfeiffer (2012)

5. Johnson, W., Vilhjalmsson, H., Marsella, S.: Serious games for language learning: How much game, how much ai. Artificial Intelligence in Education: Supporting Learning through Intelligent and Socially Informed Technology (2005) 306-313

6. deNoyelles, A., Seo, K.K.J.: Inspiring equal contribution and opportunity in a 3D multi-user virtual environment: Bringing together men gamers and women nongamers in Second Life. Computers \& Education 58(1) (2012) 21 - 29

7. Yee, N.: The demographics, motivations, and derived experiences of users of massively multi-user online graphical environments. Presence: Teleoperators and virtual environments 15(3) (2006) 309-329 
8. Jarmon, L., Traphagan, T., Mayrath, M., Trivedi, A.: Virtual world teaching, experiential learning, and assessment: An interdisciplinary communication course in Second Life. Computers \& Education 53(1) (2009) 169-182

9. Bartle, R.: From muds to mmorpgs: The history of virtual worlds. International Handbook of Internet Research (2010) 23-39

10. Duncan, I., Miller, A., Jiang, S.: A taxonomy of virtual worlds usage in education. British Journal of Educational Technology (2012)

11. Collins, C.: Looking to the future: Higher education in the Metaverse. Educause Review 43(5) (2008) 51-63

12. Seeber, K.: SIMON: An online clearing house for interpreter training materials. Technology and Teacher Education Annual 4 (2006) 2403

13. Hansen, I., Shlesinger, M.: The silver lining: Technology and self-study in the interpreting classroom. Interpreting 9(1) (2007) 95-118

14. Braun, S.: ELISA-a pedagogically enriched corpus for language learning purposes. Corpus Technology and Language Pedagogy: New Resources, New Tools, New Methods (2006)

15. Sandrelli, A., Jerez, M., et al.: The impact of Information and Communication Technology on interpreter training. The Interpreter and translator trainer $\mathbf{1}(2)$ (2007) 269-303

16. Warburton, S.: Second Life in higher education: Assessing the potential for and the barriers to deploying virtual worlds in learning and teaching. British Journal of Educational Technology 40(3) (2009) 414-426

17. Jennings, N., Collins, C.: Virtual or Virtually U: Educational Institutions in Second Life. International Journal of Social Sciences 2(3) (2007) 180-186

18. Boulos, M., Hetherington, L., Wheeler, S.: Second Life: an overview of the potential of 3D virtual worlds in medical and health education. Health Information \& Libraries Journal 24(4) (2007) 233-245

19. Ye16, E., Fang, Y., Liu18, C., Chang19, T., Dinh, H.: Appalachian tycoon: an environmental education game in second life. In: Second Life Education Workshop 2007. (2007) 72

20. Ryoo, J., Techatassanasoontorn, A., Lee, D.: Security education using Second Life. IEEE Security Privacy Magazine 7(2) (2009) 71-74

21. Ang, K., Wang, Q.: A case study of engaging primary school students in learning science by using Active Worlds. In: Proceedings of the First International LAMS Conference 2006: Designing the Future of Learning. (2006)

22. Dickey, M.: Three-dimensional virtual worlds and distance learning: two case studies of Active Worlds as a medium for distance education. British Journal of Educational Technology 36(3) (2005) 439-451

23. Prasolova-Førland, E.: Analyzing place metaphors in 3D educational collaborative virtual environments. Computers in Human Behavior 24(2) (2008) 185-204

24. Zhao, H., Sun, B., Wu, H., Hu, X.: Study on building a 3D interactive virtual learning environment based on OpenSim platform. In: Audio Language and Image Processing (ICALIP), 2010 International Conference on, IEEE (2010) 1407-1411

25. Konstantinidis, A., Tsiatsos, T., Demetriadis, S., Pomportsis, A.: Collaborative learning in opensim by utilizing sloodle. In: Telecommunications (AICT), 2010 Sixth Advanced International Conference on, IEEE (2010) 90-95

26. Savin-Baden, M., Gourlay, L., Tombs, C., Steils, N., Tombs, G., Mawer, M.: Situating pedagogies, positions and practices in immersive virtual worlds. Educational Research 52(2) (2010) 123-133 
27. Thackray, L., Good, J., Howland, K.: Learning and teaching in Virtual Worlds: Boundaries, challenges and opportunities. Researching Learning in Virtual Worlds (2010) 139-158

28. Salmon, G., Nie, M., Edirisingha, P.: Developing a five-stage model of learning in Second Life. Educational Research 52(2) (2010) 169-182

29. Carr, D., Oliver, M., Burn, A.: Learning, teaching and ambiguity in virtual worlds. In: Learning in Virtual Environments International Conference. (2008) 83

30. Moschini, E.: The Second Life Researcher Toolkit-An exploration of in-world tools, methods and approaches for researching educational projects in Second Life. Researching Learning in Virtual Worlds (2010) 31-51

31. Tymczynska, M.: Integrating in-class and online learning activities in a healthcare interpreting course using Moodle. The Journal of Specialised Translation (12) (2009) 148-164

32. Dougiamas, M., Taylor, P.: Moodle: Using learning communities to create an open source course management system. In: Proceedings of world conference on educational multimedia, hypermedia and telecommunications. Volume 3. (2003)

33. Connolly, T., Boyle, E., MacArthur, E., Hainey, T., Boyle, J.: A systematic literature review of empirical evidence on computer games and serious games. Computers \& Education (2012)

34. Zender, R., Dressler, E., Lucke, U., Tavangarian, D.: Pervasive media and messaging services for immersive learning experiences. In: Pervasive Computing and Communications PerCom 2009. IEEE International Conference on. (2009) 1 -6

35. Blais, C., Brutzman, D., Horner, D., Nicklaus, M.: Web-based 3D technology for scenario authoring and visualization: The SAVAGE project. In: Interservice/Industry Training, Simulation \& Education Conference (I/ITSEC), NTSA (2001)

36. Kemp, J., Livingstone, D., Bloomfield, P.: SLOODLE: Connecting VLE tools with emergent teaching practice in Second Life. British Journal of Educational Technology 40(3) (2009) 551-555

37. Glinka, F., Ploss, A., Gorlatch, S., Müller-Iden, J.: High-level development of multiserver online games. Int. J. Comput. Games Technol. 2008 (January 2008) $3: 1-3: 16$

38. Roberts, J.C., Gittins, R., Thomas, R.: Scale and the construction of real-world models in second life. Journal of Gaming \& Virtual Worlds 2(3) (2010) 259-279

39. Gonze, L., Friedrich, M., Kaye, R.: XML shareable playlist format version 1 (2008)

40. Hix, D., Hartson, H.: Developing user interfaces: ensuring usability through product \& process. John Wiley \& Sons (1993)

41. Brooke, J.: SUS - A quick and dirty usability scale. In: Usability Evaluation in Industry. Taylor \& Francis (1996) 189-194

42. Lindgaard, G., Chattratichart, J.: Usability Testing: What Have We Overlooked? In: Conference on Human Factors in Computing Systems (CHI '07), San Jose, California, USA, ACM Press (Apr 2007) 1415-1424

43. Scholfield, P.: Quantifying language: A researcher's and teacher's guide to gathering language data and reducing it to figures. Multilingual Matters Limited (1995)

44. Bangor, A., Kortum, P.T., Miller, J.T.: An empirical evaluation of the system usability scale. International Journal of Human-Computer Interaction 24(6) (2008) 574-594

45. Panëels, S.A., Roberts, J.C.: Review of designs for haptic data visualization. IEEE T. Haptics 3(2) (2010) 119-137 